\title{
Optical Spectroscopy Study of the Interaction Between Quercetin and Human Serum Albumin
}

\author{
T. WYBRANOWSKI* AND S. KRUSZEWSKI
}

Medical Physics Division, Biophysics Department, Collegium Medicum of Nicolaus Copernicus University Jagiellońska 13, 85-067 Bydgoszcz, Poland

Optical methods are very useful for the study on behavior of molecules in albumin-containing solutions. The interaction between quercetin (QUE) and human serum albumin (HSA) under physiological conditions was investigated by the methods of UV-Vis absorption and fluorescence spectroscopy. Fluorescence data show that enhancing of quercetin fluorescence in the presence of HSA is the result of formation of the HSA-QUE complex. On the basis of fluorescence data, the binding affinity constant of quercetin to HSA is determined. In this paper we have attempted to perform a kinetic study of the oxidation of quercetin in presence of human serum albumin by absorption spectroscopy. It has been shown that quercetin easily oxidizes at $\mathrm{pH} 7.4$. The addition of HSA to quercetin solution induces changes in the absorption spectrum. In the human serum albumin solution, the time of quercetin oxidation is longer than in the case of quercetin diluted in phosphate buffered saline. Human albumin also contributes to stabilization of quercetin. These results suggest that HSA prevents degradation of quercetin in blood.

DOI: 10.12693 /APhysPolA.125.A-57

PACS: 87.64.K-, 87.64.kv, 87.15.kp

\section{Introduction}

Serum albumin is the basic protein of blood plasma [1]. This protein interacts with drug through its binding site $[2,3]$. The interaction between drugs and serum protein has an effect on biological half-life, metabolism and excretion of drugs. Only free fraction of drug is pharmacologically active and its concentration is closely related to the therapeutic effect $[4,5]$. If the unbound fraction of drug diffuses across the wall of small arterioles, the equilibrium is disturbed and the bound fraction starts to separate from albumin. The binding of a drug and a protein may be caused by electrostatic interaction, hydrogen bonds, van der Waals interaction, or placement of the drug in hydrophobic pocket of HSA (hydrophobic interaction) [6].

In recent few years, great attention has been paid to the study of flavonoids [7]. They are known to show biological activity. One of the most prominent flavonoids is quercetin contained in vegetables, oils, and fruits. It has been reported to exert multiple biological effects as an antioxidant [8]. Flavonoids are reported to be antioxidants which neutralize free radicals by giving them electrons [9]. Analysis of quercetin oxidation is important due to its pharmacological properties. Because the quercetin strongly binds to human albumin, it is desirable to study the oxidation properties of quercetin after binding to HSA. Such interaction of quercetin with human albumin may change its antioxidant properties and some other biological effects of the flavonoid.

\section{Materials and methods}

\subsection{Materials and instrumentation}

Quercetin and human serum albumin were received from Sigma-Aldrich. Stock solution of quercetin was pre-

*corresponding author; e-mail: tomaszwybranowski@cm.umk.pl pared in ethanol. For absorption measurements, concentration of quercetin in final samples was equal to $10 \mu \mathrm{M}$. The albumin was diluted in phosphate-buffered saline (PBS) at $\mathrm{pH}$ 7.4. The spectrophotometer UV-Vis JASCO V-550 was used for recording absorption spectra of quercetin and other solutions. The kinetic studies of the oxidation of quercetin both in absence and presence of human serum albumin consisted of a single series of measurements. Temperature of the samples was kept constant $\left(37^{\circ} \mathrm{C}\right)$ using TW2.03 (ELMI) ultrathermostat. For fluorescence excitation of quercetin, the radiation of $450 \mathrm{~nm}$ wavelength was applied. Fluorescence spectra were obtained on HR4000 Spectrometer.

\subsection{Methods of affinity determination}

For determining the binding constant of quercetin to HSA, the following formula was used [10]:

$$
K=\frac{\left[H S A_{\mathrm{B}}\right]}{\left[C_{\mathrm{F}}\right]\left[H S A_{\mathrm{F}}\right]},
$$

where $H S A_{\mathrm{B}}$ is the concentration of bound protein, while $C_{\mathrm{F}}$ and $H S A_{\mathrm{F}}$ are concentrations of free dye and free protein, respectively. Because $H S A_{\mathrm{F}}=H S A_{\mathrm{T}}-H S A_{\mathrm{B}}$, from Eq. (1) one can obtain

$$
\frac{H S A_{\mathrm{B}}}{H S A_{\mathrm{T}}}=\frac{K C_{\mathrm{F}}}{1+K C_{\mathrm{F}}},
$$

where $H S A_{\mathrm{T}}$ is the total concentration of HSA.

Due to the enhancement of fluorescence emission of quercetin after binding drug to HSA, it can be deduced that

$$
\frac{H S A_{\mathrm{B}}}{H S A_{\mathrm{T}}}=\frac{\Delta F}{\Delta F_{\max }},
$$

where $\Delta F$ is the difference between fluorescence of HSAQUE complex and fluorescence of free quercetin and $\Delta F_{\max }$ is the maximum fluorescence corresponding to the saturation.

The $C_{\mathrm{F}}$ value was obtained using relationship

$$
C_{\mathrm{F}}=C-H S A_{\mathrm{B}} \text {, }
$$


Equation (4) can be rewritten as

$$
C_{\mathrm{F}}=C-\frac{H S A_{\mathrm{T}}}{H S A_{\mathrm{T}}} H S A_{\mathrm{B}} \text {. }
$$

By substituting Eq. (3) into Eq. (5), the following formula can be obtained

$$
C_{\mathrm{F}}=C-\frac{\Delta F}{\Delta F_{\max }} H S A_{\mathrm{T}} .
$$

Finally, by rearranging Eq. (1) and from Eq. (6) one can obtain

$$
\frac{1}{\Delta F}=\frac{1}{\Delta F_{\max }}+\frac{1}{K \Delta F_{\max }} \frac{1}{C-\frac{\Delta F}{\Delta F_{\max }} H S A_{\mathrm{T}}} .
$$

This equation is known as the Klotz equation.

Equations (1) and (6) can be also rearranged yielding the modified Scatchard equation

$$
\frac{\Delta F / \Delta F_{\max }}{C-\frac{\Delta F}{\Delta F_{\max }} H S A_{\mathrm{T}}}=-K \frac{\Delta F}{\Delta F_{\max }}+K .
$$

The ordinate intercept of the regression line $\frac{\Delta F / \Delta F_{\max }}{C-\left(\Delta F / \Delta F_{\max }\right) H S A_{\mathrm{T}}}$ on $\Delta F / \Delta F_{\max }$ can be interpreted as $K$. By measuring the fluorescence emission $\Delta F$ as a function of the quercetin concentration $C$, finding the maximum value of HSA-quercetin complex fluorescence emission $\Delta F_{\max }$, and using Eq. (7) or $(8)$, it is possible to determine the binding constant $K$ of drug to albumin. The single fluorescence emission spectra for each quercetin concentration were recorded. Correlation coefficients and standard errors of slopes and intercepts were calculated with the use of ORIGIN 7.0 software application. Then, standard error of the binding constant $K$ was estimated.

\section{Results and discussion}

In the presented work, the rate of the oxidation reaction of quercetin at $\mathrm{pH} 7.4$ was determined. In Fig. 1, absorption spectra of quercetin recorded at different instants of time are shown. Quercetin exhibits two ma-

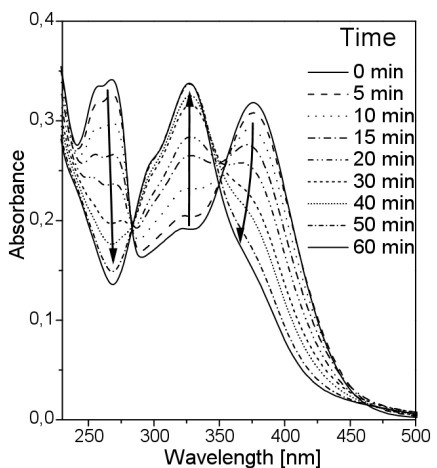

Fig. 1. Oxidation of quercetin in PBS ( $\mathrm{pH}$ 7.4). Changes in absorbance spectrum in time. Direction of the arrows shows the order of the recorded spectra in time.

jor absorption bands in the UV-Vis region, namely at $376 \mathrm{~nm}$ and $268 \mathrm{~nm}$. During a series of measurements, the absorbance at $376 \mathrm{~nm}$ decreased and was replaced<smiles>O=C1C=CC(C2=C(O)C(O)c3c(O)cc(O)cc3O2)=CC1=O</smiles>

Fig. 2. The molecular structure of quercetin and quinone.

by a new peak with absorbance at $327 \mathrm{~nm}$. Simultaneously, the absorbance at the wavelength of $268 \mathrm{~nm}$ with a shoulder at $257 \mathrm{~nm}$ decreased. The maximum of absorption at $327 \mathrm{~nm}$ increased with time until 60 minutes which indicates that certain time was needed to complete oxidation reactions. After that time, all quercetin molecules were transformed into new molecules called quinone (Fig. 2). This process occurs in two steps. In the beginning, two electrons and two hydrogen atoms are abstracted from quercetin. The second step is reorientation of the molecule of quercetin. The new structure is unstable and the degradation products appear [11] Time of conversion of quercetin into quinone is called the deprotonation time. This time strongly depends on surrounding medium and on the solution's pH. After addition of free radicals to quercetin solution, the deprotonation time is reduced. Free radicals are atoms with unpaired number of electrons. The antioxidant property of flavonoids can be characterized by measuring the deprotonation time or determining numbers of free radicals scavenged by flavonoid molecules.

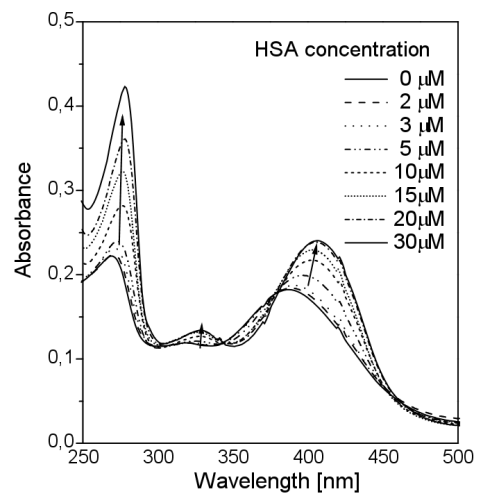

Fig. 3. Absorbance spectra of quercetin in presence of various concentration of HSA $(0-30 \mu \mathrm{M})$. Direction of the arrows shows the increase of HSA concentration. All experiments were conducted in PBS at $\mathrm{pH} 7.4$, temperature $37^{\circ} \mathrm{C}$, and quercetin concentration $10 \mu \mathrm{M}$.

Figure 3 shows the absorbance spectra of quercetin with different concentration of human serum albumin diluted in PBS at $\mathrm{pH}$ 7.4. It can be seen that the absorbance spectrum of quercetin in presence of HSA is redshifted in comparison to the spectrum of free quercetin. It is the evidence of binding of quercetin to human serum albumin. The visible absorption peak shifts by about 30 


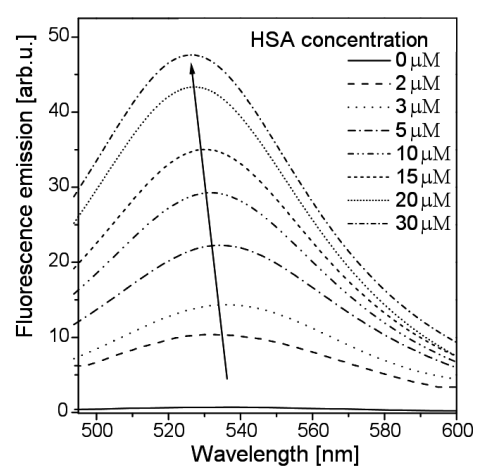

Fig. 4. Fluorescence spectra of quercetin in presence of various concentration of HSA $(0-30 \mu \mathrm{M})$. Direction arrow shows the increase of HSA concentration. All experiments were conducted in PBS at $\mathrm{pH} 7.4$ and temperature of $37^{\circ} \mathrm{C}$ using quercetin concentration $10 \mu \mathrm{M}$.

$\mathrm{nm}$, from $376 \mathrm{~nm}$ to $406 \mathrm{~nm}$. This band is characteristic for the formation of a HSA-quercetin complex. After adding HSA, the ultraviolet band with a maximum between $269 \mathrm{~nm}$ and $278 \mathrm{~nm}$ strongly increases. Simultaneously, a little increase in the absorbance is observed at $328 \mathrm{~nm}$. One can see that when concentration of HSA is higher than $20 \mu \mathrm{M}$, the absorption spectra between 300 $\mathrm{nm}$ and $500 \mathrm{~nm}$ do not change. However, the ultraviolet peak increases. Such a difference in absorption behavior can be attributed to different binding of quercetin to HSA. It may be related to binding of quercetin to other binding site of HSA when the concentration of HSA grows significantly.

The fluorescence spectrum of quercetin diluted in PBS ( $\mathrm{pH} 7.4$ ) with excitation at $450 \mathrm{~nm}$ exhibits a very low band with maximum at $535 \mathrm{~nm}$. After adding human serum albumin, the band strongly increases as shown in Fig. 4. This suggests that the surrounding around of quercetin changes. The changes which appear in fluorescence spectra in the presence of HSA provide information about binding affinity of quercetin to albumin.

Taking the advantage of the fact that value of fluorescence emission depends on concentration of quercetin, fluorescence spectra analysis can be used for determination of affinity constant of the drug to HSA. The drug's binding constant can be determined by measurements of the fluorescence emission of quercetin, because the fraction of occupied protein is proportional to fluorescence emission of quercetin. Fig. 5A shows the fluorescence emission as a function of quercetin concentration. Next, as it is described in Sec. 2 according to Eq. (7), the double-reciprocal plots were drawn and the associated constant was calculated. It is seen that the inverse of fluorescence emission of HSA-quercetin complex is a linear function of inverse of free quercetin concentration. Slope of the line fitted to points $\left(1 / C_{\mathrm{Fi}}, 1 / \Delta F_{\mathrm{i}}\right)$ determine $1 /\left(\Delta F_{\max } K\right)$ according to Eq. (7). The maximum fluorescence emission estimated on the basis of fluorescence spectra is equal to 50 . We have found that the association constant of quercetin to albumin was equal to $2.54 \pm 0.04 \times 10^{5} \mathrm{M}^{-1}$. To estimate the binding constant, the regression analysis of $\Delta F /\left(\Delta F_{\max } C_{\mathrm{F}}\right)$ versus $\Delta F / \Delta F_{\max }$ according to Eq. (8) was also used. The obtained value was $2.38 \pm 0.1 \times 10^{5} \mathrm{M}^{-1}$. The binding constant $K$ has been determined at $37^{\circ} \mathrm{C}$, and was slightly larger than the one obtained at $25^{\circ} \mathrm{C}$ in the seminal paper by Sengupta and Sengupta [7]. The standard Gibbs free energy change of binding quercetin to albumin was found to be approximately $-32 \mathrm{~kJ} / \mathrm{mol}$ at $37^{\circ} \mathrm{C}$. Due to high affinity of QUE to HSA, binding to HSA must have an effect on oxidation and stabilization of quercetin.
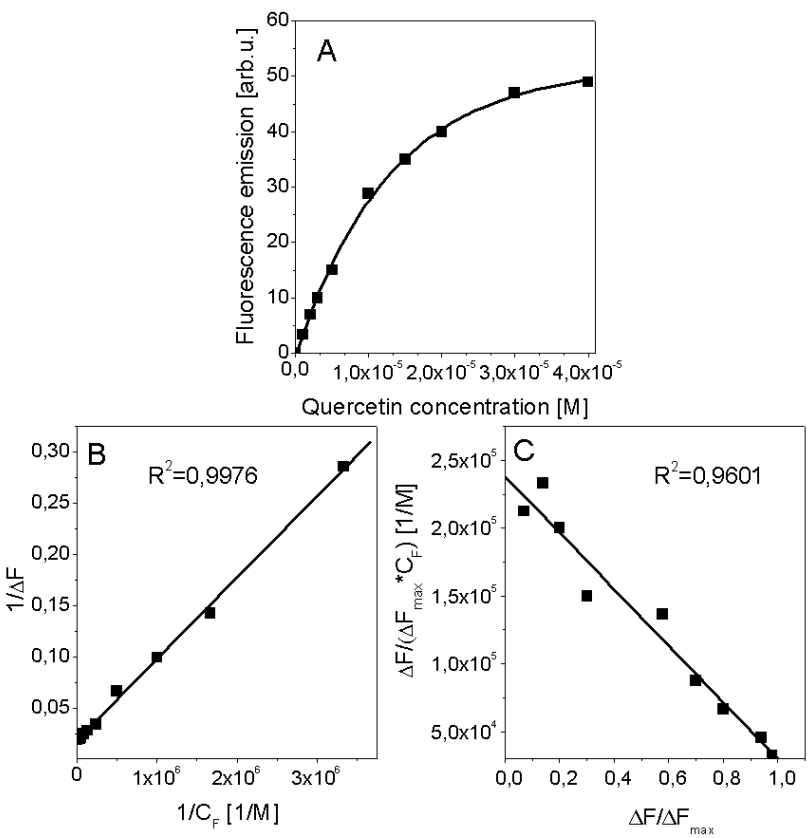

Fig. 5. Enhancements of quercetin fluorescence as a function of quercetin concentration (graph A). Graph B shows double reciprocal plot for quercetin binding with HSA. Graph C is the Scatchard's plot obtained according to Eq. 8. All experiments were conducted in PBS at $\mathrm{pH} 7.4$ and temperature of $37^{\circ} \mathrm{C}$, using HSA with concentration of $10 \mu \mathrm{M}$.

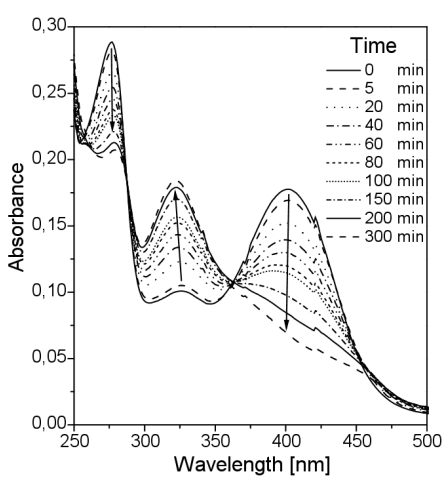

Fig. 6. Oxidation of quercetin in presence of human serum albumin. The HSA/quercetin molar ratio was: $1: 1$. Direction of the arrows points out the changes in time. Arrows indicate direction of the absorbance spectrum change in time. 


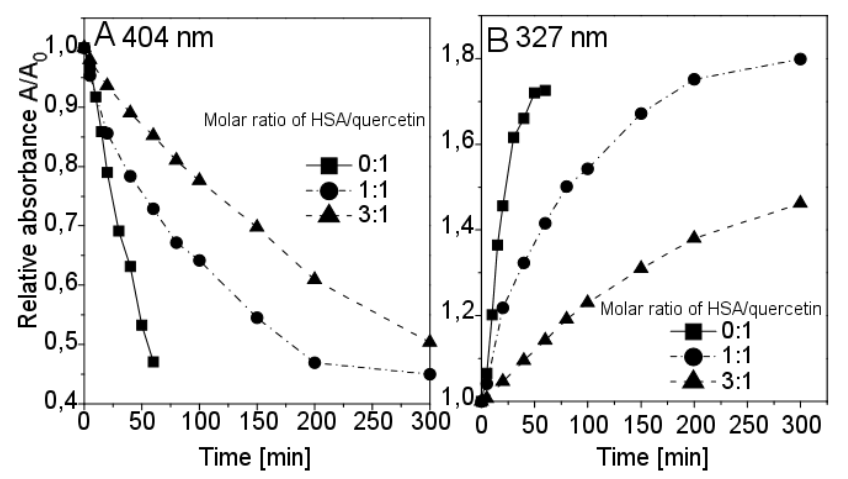

Fig. 7. Relative absorbance of quercetin as a function of incubation time in the presence of HSA for two wavelengths, $404 \mathrm{~nm} \mathrm{(A)} \mathrm{and} 327 \mathrm{~nm}$ (B) for HSA/quercetin molar ratios of 0:1 (solid line $\mathbf{-}$ ), 1:1 (dash-dotted line $\bullet$ ), and 3:1 (dashed line $\boldsymbol{\Delta}$ ). $\mathrm{A}_{\mathrm{o}}$ is the absorbance of free quercetin in PBS ( $\mathrm{pH}$ 7.4).

Figure 6 shows the absorbance spectra of HSAquercetin complex at molar ratio 1:1 at different stages of the experiment. The absorbance spectra changed with the increase of time. The decrease of peaks at $404 \mathrm{~nm}$ and $278 \mathrm{~nm}$ was paralleled by the increase in absorbance at $327 \mathrm{~nm}$. It be should noticed that absorption of quercetin in presence of HSA changed slower than absorption observed for free quercetin in pure PBS. After $300 \mathrm{~min}$, absorption spectra of the complex did not change and the degradation process was not observed. The absorbance at $278 \mathrm{~nm}$ for complex after $300 \mathrm{~min}$ was higher in comparison to maximum of absorption at $278 \mathrm{~nm}$ of quercetin diluted in PBS solution which indicates that quinone quercetin was still binding to HSA. As the degradation products did not appear after transformation of quercetin into quinone, this may be of a significance for oxidation properties of quercetin. Some authors claim that the degradation products can transfer hydrogen and electrons to free radicals but this process is less efficient than abstraction of the hydrogen atom from quercetin [12]. This showed that quercetin in solution of human serum albumin still retained its antioxidant activity.

Next, kinetic measurements of quercetin at wavelengths of $404 \mathrm{~nm}$ and $327 \mathrm{~nm}$ were studied in solution containing HSA (Fig. 7A, B). The measurements of absorbance spectra at these wavelengths at two concentration of HSA have been analyzed. By comparing the deprotonation time of HSA-quercetin complex with this of quercetin it can be found that the deprotonation time of HSA-quercetin complex is shorter. It may prove that oxidation process of quercetin is less efficient in the presence of HSA. In the sample where the concentration of protein was three times higher, the deprotonation time had increased more than five times. It is seen that the shape of the kinetic spectra differ for different molar ratio of HSA and quercetin. The observed changes in absorption spectra of quercetin with different concentration of HSA may be caused by changes in the environment of quercetin.
For high concentration of HSA, quercetin molecules may be isolated from water in hydrophobic binding pockets contained in HSA [13]. In consequence, the oxidation process is inhibited. It suggests that quercetin in blood can be more stable and can keep longer the ability to reduce free radicals in comparison to quercetin in PBS.

\section{Conclusions}

The antioxidant effect of quercetin might be attributed to their properties of scavenging free-radical species. Measurements of absorption spectra provide important information about oxidation capacity of quercetin. In the present study, attention was paid mainly to oxidation time of quercetin as a key to understanding the behavior of quercetin in blood. Many experiments were undertaken to study the absorbance spectra of quercetin in presence of human serum albumin. A simple method based on measurements of fluorescence emission of quercetin in presence HSA allowed to determine the affinity constant of quercetin to albumin. The presented results show that quercetin exhibits high affinity to HSA. Formation of quercetin-HSA associates leads to the redshift of the absorption spectra. The time of oxidation of quercetin was strongly influenced by HSA concentration. As expected, the deprotonation time of quercetin decreases as HSA concentration increases. This paper provides evidence that albumin prevents quercetin oxidation. The presented results suggest that binding of quercetin to HSA have considerable significance when it comes to the pharmacological effects of flavonoids.

\section{References}

[1] U. Kragh-Hansen, V.T.G. Chuang, M. Otagiri, Biol. Pharm. Bull. 25, 695 (2002).

[2] C. Bertucci, V. Andrisano, R. Gotti, J. Chromatogr. B 768, 147 (2002).

[3] G. Sudlow, D.J. Birkett, D.N. Wade, Mol. Pharmacol. 11, 824 (1975).

[4] Y. Ni, S. Su, S. Kokot, Anal. Chim. Acta 580, 206 (2006).

[5] H. Mandula, J.M. Parepally, R. Feng, Q.R. Smith, J. Pharmacol. Exp. Ther. 317, 667 (2006).

[6] U. Kragh-Hansen, F. Hellec, B. de Foresta, M. le Maire, J.V. Møller, Biophys. J 80, 2898 (2001).

[7] B. Sengupta, P.K. Sengupta, Biochem. Biophys. Res. Commun. 299, 400 (2002).

[8] C.D. Kanakis, P.A. Tarantilis, M. Polissiou, S. Diamantoglou, H.A. Tajmir-Riahi , J. Mol. Struct. 798 , 69 (2006).

[9] S.B. Bukhari, S. Memon, M. MahroofTahir, M.I. Bhanger, Spectrochim. Acta A Mol. Biomol. Spectrosc. 71, 1901 (2009).

[10] B. Mishra, A. Barik, I.K. Priyadarsini, H. Mohan, J. Chem. Sci. 117, 641 (2005).

[11] O. Dangles, C. Dufour, S. Bret, J. Chem. Soc., Perkin Trans. 2, 737 (1999).

[12] O. Dangles, G. Fargeix, C. Dufour, J. Chem. Soc., Perkin Trans. 2, 1387 (1999).

[13] B. Ziomkowska, S. Kruszewski, M. Cyrankiewicz, Comb. Chem. High Thr. Screen 10, 459 (2007). 